\title{
Compatibility of a novel filter paper-based bio-safe sputum transport kit with line probe assay for diagnosing drug-resistant tuberculosis: a single-site evaluation study
}

\author{
Divya Anthwal ${ }^{1,2}$, Rakesh Kumar Gupta ${ }^{1,2}$, Ritu Singhal ${ }^{3}$, Manpreet Bhalla ${ }^{3}$, Ajoy Kumar Verma ${ }^{3}$, \\ Khalid Umar Khayyam ${ }^{3}$, Vithal Prasad Myneedu ${ }^{3}$, Rohit Sarin ${ }^{3}$, Ashawant Gupta ${ }^{4}$, Nalini Kant Gupta ${ }^{4}$, \\ Manjula Singh ${ }^{5}$, Jaya Sivaswami Tyagi $\mathbb{B}^{2,6}$ and Sagarika Haldar $\mathbb{1}^{1,2}$
}

\begin{abstract}
${ }^{1}$ Dept of Experimental Medicine and Biotechnology, Postgraduate Institute of Medical Education and Research, Chandigarh, India. ${ }^{2}$ Translational Health Science and Technology Institute, NCR Biotech Science Cluster, Faridabad, India. ${ }^{3}$ Dept of Microbiology, National Institute of Tuberculosis and Respiratory Diseases, New Delhi, India. ${ }^{4}$ Advanced Microdevices Pvt Ltd, Ambala Cantt, India. ${ }^{5}$ India TB Research Consortium, Indian Council of Medical Research, New Delhi, India. ${ }^{6}$ Dept of Biotechnology, All India Institute of Medical Sciences, New Delhi, India.
\end{abstract}

Corresponding author: Sagarika Haldar (sagarikahaldar.pgimer@gmail.com)
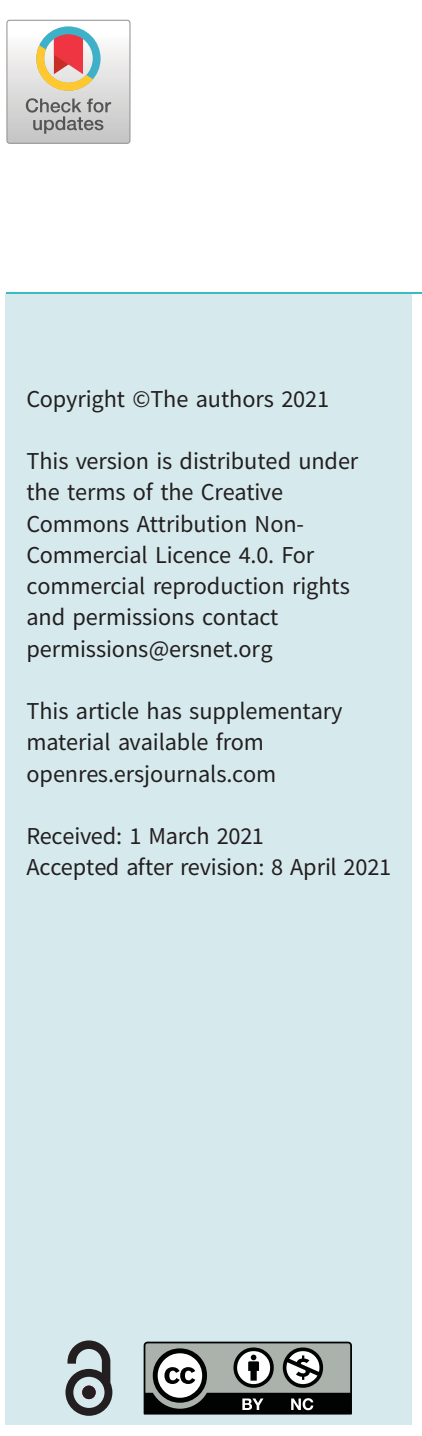

Shareable abstract (@ERSpublications)

The adoption of bio-safe "TB Concentration \& Transport" kit by Microscopy Centres can potentially overcome the challenge of transporting infectious sputum to central laboratories and provide universal DST services to TB subjects residing in remote areas. https://bit.ly/2QrQ5qL

Cite this article as: Anthwal D, Gupta RK, Singhal R, et al. Compatibility of a novel filter paper-based bio-safe sputum transport kit with line probe assay for diagnosing drug-resistant tuberculosis: a single-site evaluation study. ERJ Open Res 2021; 7: 00137-2021 [DOI: 10.1183/23120541.00137-2021].

\section{Abstract}

Background Near-patient access to appropriate tests is a major obstacle for the efficient diagnosis of tuberculosis (TB) and associated drug resistance.

Methods We recently developed the "TB Concentration \& Transport” kit for bio-safe, ambient-temperature transportation of dried sputum on Trans-Filter, and the "TB DNA Extraction” kit for DNA extraction from Trans-Filter for determining drug resistance by DNA sequencing. In the present study, we evaluated the compatibility of Kit-extracted DNA with Hain's line probe assays (LPAs), which are endorsed by National TB programmes for the detection of drug resistance in sputum collected from presumptive multidrugresistant TB patients $(n=207)$.

Results Trans-Filter-extracted DNA was seamlessly integrated with the LPA protocol (Kit-LPA). The sensitivity of Kit-LPA for determining drug resistance was 83.3\% for rifampicin (95\% CI 52-98\%), 77.7\% for isoniazid (95\% CI 52-94\%), 85.7\% for fluoroquinolones (95\% CI 42-100\%) and 66.6\% for aminoglycosides (95\% CI 9-99\%), with a specificity range of 93.7\% (95\% CI 87-97) to 99.1\% (95\% CI 95-100) using phenotypic drug susceptibility testing (DST) as a reference standard. A high degree of concordance was noted between results obtained from Kit-LPA and LPA (99\% to 100\% ( $\kappa$ value: 0.83-1.0)).

Conclusions This study demonstrates successful integration of our developed kits with LPA. The adoption of these kits across Designated Microscopy Centres in India can potentially overcome the existing challenge of transporting infectious sputum at controlled temperature to centralised testing laboratories and can provide rapid near-patient cost-effective "Universal DST" services to TB subjects residing in remote areas.

\section{Introduction}

Tuberculosis (TB) is one of the leading causes of death worldwide and India accounts for $26 \%$ of the world's total TB burden [1]. Around 500000 new cases of rifampicin (RIF)-resistant TB were noted in 2019, of which 78\% were multidrug-resistant TB (MDR-TB) [1]. The rapid increase in the number of drug-resistant TB cases has been further exacerbated by the ongoing coronavirus disease 2019 (COVID-19) pandemic, which has created critical gaps in diagnosing and providing care to TB patients [2]. 
This highlights the necessity of widespread drug susceptibility testing (DST) for implementing patient-centric anti-TB regimens [1]. However, DST implementation is a major challenge in primary healthcare centres (PHCs), particularly in remote geographical areas of India and other high-burden countries, where DST facilities are restricted to centralised laboratories such as National/Intermediate reference laboratories (NRLs/IRLs). At present, DST services are extended to patients residing in remote areas by sample transport under temperature-controlled and bio-safe containment conditions [3]. In view of these impediments, a safe and robust modality for sputum transportation from Designated Microscopy Centres (DMCs) or District Tuberculosis Centres to centralised laboratories is a priority requirement of the National TB Elimination Programme (NTEP) [3].

The technology for the detection of drug-resistant TB is moving away from conventional culture-based DST approaches towards rapid molecular DST (Mol-DST). The World Health Organization (WHO)-endorsed tests for drug resistance testing include the Xpert ${ }^{\circledR}$ MTB/RIF assay (Xpert (Cepheid, Sunnyvale, USA)), Xpert ${ }^{\circledR}$ MTB/RIF Ultra assay (Cepheid, Sunnyvale, USA), Truenat ${ }^{\mathrm{TM}}$ MTB-Rif Dx assay (Molbio Diagnostics, Goa, India) and line probe assays (LPA) [4]. At present, Xpert and Truenat tests provide information only on rifampicin resistance, while LPA, namely GenoType ${ }^{\circledR}$ MTBDRplus VER 2.0 (first-line LPA) and GenoType ${ }^{\circledR}$ MTBDRsl tests VER 2.0 (second line LPA), both from Hain Lifesciences, Nehren, Germany, are comprehensive molecular tests for MDR-TB and extensively drugresistant TB (XDR-TB), respectively. In India, Hain's LPAs (henceforth referred to as LPA) are mainly used in the NTEP programme and are recommended for use in only direct smear-positive sputum specimens and culture isolates of smear-negative sputum samples [5]. In India in 2019, 346282 first-line LPA and 72748 second-line LPA tests were performed, compared to only 16399 culture-DST tests [3]. This indicates the scalability of LPA testing. However, the use of LPA is restricted to NRLs/IRLs and certified laboratories $(n=64)$ with sophisticated facilities and trained manpower that are not available at the DMC level [3], which raises the logistic challenge of transporting infectious sputum from remote areas to the testing laboratory and also poses bio-safety issues.

To address this unmet need, we have recently developed the "TB Concentration \& Transport” kit for collecting bacteria present in sputum on a Trans-Filter device and "TB DNA Extraction” kit for DNA extraction from Trans-Filter [6]. This bio-safe Trans-Filter can be shipped at ambient temperature, and DNA can be extracted at the DST laboratory [6] using the "TB DNA Extraction” kit (supplementary figure S1). The primary objectives of this study were to evaluate the compatibility of Kit-extracted DNA with LPA (Kit-LPA) and to compare the performance (diagnostic accuracy) of Kit-LPA with that of the WHO-endorsed LPA test. The secondary objectives of the study were to evaluate the bio-safety of the Trans-Filter device and to obtain performance feedback from the scientists and technicians who have used the kits.

\section{Materials and methods}

\section{Study subjects and design}

This study was designed and supervised by Translational Health Science and Technology Institute (THSTI), Faridabad, and the All India Institute of Medical Sciences (AIIMS), New Delhi. Presumptive MDR-TB/XDR-TB patients were included in the study according to the Programmatic Management of Drug-Resistant TB Guidelines, namely belonging to one or more of the following categories: TB patients found positive on any follow-up sputum smear examination during treatment with first-line drugs, including treatment failures, drug-resistant TB patients' contacts, previously treated TB patients, recurrent TB patients (TB diagnosed after completing a course of TB treatment) and patients retrieved after loss to follow-up [5]. All patients were enrolled after Institutional Ethical Clearance at the National Institute of Tuberculosis and Respiratory Diseases (NITRD, NITRD/EC/2017/0228) and THSTI (THS 1.8.1/(70)). Sample size $(n=234)$ was estimated based on $85 \%$ power, $\alpha$ of $5 \%$ and positivity of $44 \%$ versus $32 \%$ of Kit-extracted DNA-based sequencing versus Mycobacteria Growth Indicator Tube (MGIT)-DST for determination of MDR-TB (unpublished data). The study was performed in a double-blind manner from June 2018 through February 2019 on prospectively collected fresh sputum samples in the Outpatient Department (OPD) at NITRD (appendix S1). This study adhered to the Standards for Reporting of Diagnostic Accuracy (STARD) guidelines, and a completed checklist is included in appendix S2.

\section{Sample collection and processing}

One sputum sample was collected from each patient. Firstly, a loopful of sputum from this sample was used to perform Ziehl-Neelsen (ZN) staining at the OPD; smears were observed and graded as recommended by NTEP guidelines [7]. Only smear-positive patients were enrolled in the study, as NTEP recommends direct LPA testing only on smear-positive sputum (figure 1). All smear-positive samples were transported to the Microbiology Department of NITRD where an aliquot of sputum was processed using 


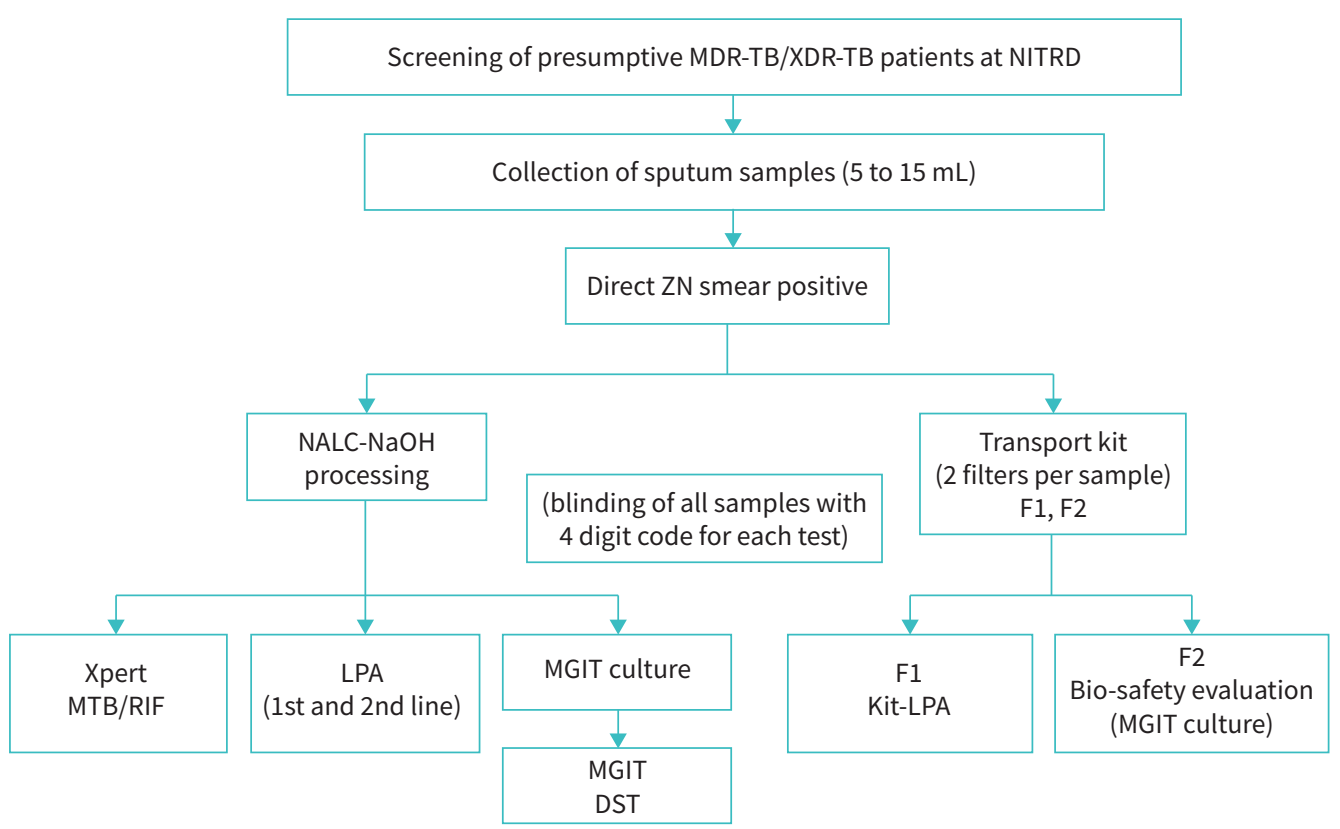

FIGURE 1 Workflow of the study. Kit-LPA: Kit-extracted DNA with line probe assay; LPA: line probe assay; MGIT: mycobacteria growth indicator tube; DST: drug susceptibility testing; MDR-TB: multidrug-resistant TB; XDR-TB: extensively drug-resistant TB; NITRD: National Institute of Tuberculosis and Respiratory Diseases; ZN: Ziehl-Neelsen stain; NALC-NaOH: N-acetyl L-cysteine-sodium hydroxide; Xpert MTB/RIF (Cepheid).

the “TB Concentration \& Transport” kit followed by LPA using DNA extracted from Trans-Filter using the "TB DNA Extraction” kit (Kit-LPA). Another aliquot was processed for bio-safety assessment as described below. The leftover sputum sample was processed by the N-acetyl L-cysteine (NALC)-sodium hydroxide $(\mathrm{NaOH})$ method for LPA, Xpert and MGIT culture in a double-blind manner. A unique 4-digit code was assigned to each sample for different tests (four codes per sputum sample); each test was performed by separate laboratory personnel. The results were decoded and analysed at the completion of the study.

\section{Line probe assay}

All sputum samples were decontaminated by the NALC-NaOH method [8]. DNA was extracted from deposits obtained after decontamination using GenoLyse ${ }^{\circledR}$ DNA Extraction Kit VER 1.0 (Hain Lifesciences, Nehren, Germany) followed by PCR amplification and reverse hybridisation (using GT-Blot 48 system, Hain Lifesciences) using first line LPA and second line LPA as per the manufacturer's instructions $[9,10]$.

Kit-LPA

All sputum samples were processed using the "TB Concentration \& Transport” kit [6]. Briefly, $400 \mu \mathrm{L}$ of "Dissolving solution" was added to $100 \mu \mathrm{L}$ of sputum and incubated for $30 \mathrm{~min}$ at room temperature. Thereafter, $300 \mu \mathrm{L}$ of liquefied sputum (equivalent to $\sim 60 \mu \mathrm{L}$ of neat sputum) was filtered through the Trans-Filter followed by the addition of "Sterilizing solution" and "Stabilizing solution” [6]. Then DNA was extracted from the Trans-Filter, which had been stored at room temperature for 2-5 days (figure 1, filter F1), using the "TB DNA Extraction" kit [6]. Kit-extracted DNA was directly used in PCR amplification followed by reverse hybridisation steps of LPA as per the manufacturer's instructions [9, 10].

\section{Xpert MTB/RIF assay}

The NALC-NaOH processed sputum samples were also subjected to Xpert assay. Briefly, $0.5 \mathrm{~mL}$ of processed sputum sample was taken and $1.5 \mathrm{~mL}$ of sample reagent was added, which was vortexed and then incubated for $15 \mathrm{~min}$ at room temperature. Then $1 \mathrm{~mL}$ of this suspension was used for Xpert [11].

\section{MGIT culture}

All decontaminated sputum samples were subjected to MGIT culture (figure 1). MGIT tubes (Becton, Dickinson, USA) showing positive signal were subjected to ZN staining (for the presence of cords) for 
presumptive detection of Mycobacterium tuberculosis (M. tuberculosis) complex. The presence of M. tuberculosis was confirmed by SD BIOLINE TB Ag MPT64 kit Rapid test (Standard Diagnostics, South Korea).

\section{MGIT-DST}

DST was performed for all M. tuberculosis culture-positive samples. Briefly, $0.5 \mathrm{~mL}$ MGIT-positive M. tuberculosis culture was inoculated into MGIT containing different drugs, i.e. RIF $\left(1 \mu \mathrm{g} \cdot \mathrm{mL}^{-1}\right)$, isoniazid (INH, $0.1 \mu \mathrm{g} \cdot \mathrm{mL}^{-1}$ ), levofloxacin (fluoroquinolone (FLQ), $1 \mu \mathrm{g} \cdot \mathrm{mL}^{-1}$ ) or kanamycin (aminoglycoside (AMN), $2.5 \mu \mathrm{g} \cdot \mathrm{mL}^{-1}$ ) as per WHO guidelines [12, 13].

\section{Bio-safety evaluation}

Bio-safety assessment culture was performed on 207 smear-positive sputum samples. All sputum samples were processed using the "Transport kit" as described previously [6]. Briefly, $400 \mu \mathrm{L}$ of "Dissolving solution" was added to $100 \mu \mathrm{L}$ of sputum and incubated for $30 \mathrm{~min}$ at room temperature. Thereafter, $300 \mu \mathrm{L}$ of liquefied sputum (equivalent to $\sim 60 \mu \mathrm{L}$ of neat sputum) was filtered through the Trans-Filter followed by the addition of "Sterilizing solution" and "Stabilizing solution". The Trans-Filter was taken out from the device using forceps and the membrane was placed into a MGIT culture tube. The MGIT culture tubes were incubated at $37^{\circ} \mathrm{C}$ for up to 42 days (figure 1, filter F2).

\section{Feedback questionnaire}

Briefly, based on feedback obtained from the users during development and pilot evaluation of these kits [6], we prepared two structured questionnaires to collect feedback from the evaluating site (NITRD, New Delhi) in the present study.

The questionnaire for the scientist was aimed at: (i) collecting information about the kits' packaging and kits' components (whether the kits included all the components in proper condition without any leakage and with proper labelling at the time of receiving); (ii) collecting viewpoints on the user manual (whether it is simple and easy to understand and descriptive enough to follow or any improvement is required); and (iii) obtaining feedback on ease of use of the kits, its user friendliness, benefits and disadvantages, and suggestions for further improvement of the kits. The questionnaire for the technician included items to obtain their viewpoint on training (for the use of kits), the user manual and ease of use of kits and their feedback on improvement in the kits, if required (appendix S3).

\section{Kit performance and statistical analysis}

Data from all presumptive MDR-TB subjects were collected using the form prepared for the study (appendix S4). Samples with incomplete data and invalid and indeterminate results were excluded from the study (invalid and indeterminate results were defined as per manufacturer's instructions [9, 11]). The analysis was done at three levels for drug resistance detection (figure 2). (1) Comparison of Kit-LPA/LPA against MGIT-DST. In this analysis, we excluded results that were TB negative by Kit-LPA/LPA and/or culture negative by MGIT and "Indeterminate" results for the respective drug targets in Kit-LPA/LPA and/ or samples with missing MGIT-DST results due to culture contamination (supplementary figures S2 and S3). (2) Comparison of Kit-LPA against LPA. In this analysis, we excluded results that were TB negative in either or both LPA and Kit-LPA and "Indeterminate" results for the respective drug targets by LPA and/

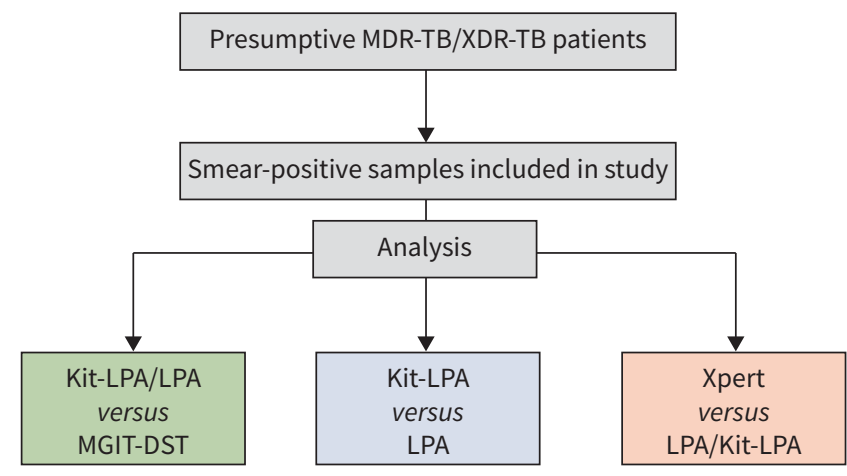

FIGURE 2 Workflow of sample analysis in the study. Kit-LPA: Kit-extracted DNA with line probe assay; LPA: line probe assay; MGIT: Mycobacteria Growth Indicator Tube; DST: drug susceptibility testing; MDR-TB: multidrugresistant TB; XDR-TB: extensively drug-resistant TB; Xpert: Xpert MTB/RIF (Cepheid). 
or Kit-LPA (supplementary figure S4). (3) Comparison of Kit-LPA/LPA against Xpert. In this analysis, we excluded results that were negative by Xpert and/or TB negative by Kit-LPA/LPA, and "Indeterminate" results for RIF resistance in Xpert and/or LPA/Kit-LPA (supplementary figure S5).

The sensitivity of Kit-LPA was calculated as (true positives)/(true positives+false negatives), where true positives were defined as samples identified as drug resistant by both Kit-LPA and phenotypic DST, and false negatives are samples that were missed by Kit-LPA but scored as resistant by phenotypic DST. Specificity was defined as (true negatives)/(true negatives+false positives), where true negatives are samples that were sensitive by both Kit-LPA and phenotypic DST, and false positives were samples showing mutations by Kit-LPA but called as sensitive by phenotypic DST. Sensitivity and specificity estimates of LPA and Xpert were also calculated similarly. Concordance between Kit-LPA and LPA results was calculated as (true positives+true negatives)/(total number of samples), and the degree of concordance/ agreement was measured by Cohen's $\kappa$ (https://www.graphpad.com/quickcalcs/kappa1/). McNemar's chi-square test was used to compare the performance of Kit-LPA versus LPA (https://epitools.ausvet.com. $\mathrm{au} /$ mcnemar). Sample size was estimated using G*Power 3 software [14].

\section{Results}

\section{Study participants}

Three hundred and twenty-nine participants were screened in the present study, of which 207 subjects who were smear-positive were enrolled in the study (figure 2). They were in the age range of 4-97 years (including 16 children, aged between 3 and 17 years), and around 69\% (144 out of 207) were males. The most common clinical symptoms were cough ( 95\%, 197 out of 207), weakness ( 90\%, 187 out of 207), loss of appetite (83\%, 172 out of 207), weight loss ( $~ 80 \%, 165$ out of 207$)$ and fever ( $61 \%, 127$ out of 207, supplementary table S1). The HIV status was available for 8 out of 207 patients, all of whom were HIV-negative.

\section{Performance of LPA and Kit-LPA versus MGIT-DST}

The detection of wild-type/mutant alleles of drug resistance genes by Kit-LPA and LPA was assessed using MGIT-DST as a gold standard (figure 3). The sensitivity of Kit-LPA was 83.3\% (95\% CI 52-98\%) and 77.7\% (95\% CI 52-94\%) for detecting RIF and INH resistance, respectively; and was quite comparable to that of LPA, which was 83.3\% for both RIF (95\% CI 52-98\%) and INH (95\% CI 59-96\%) resistance (table 1). For detecting resistance to FLQs and AMNs, the sensitivity of both Kit-LPA and LPA was 85.7\% (95\% CI $42-100 \%$ ) and 66.6\% (95\% CI 9-99\%), respectively (table 1). The specificity range was quite similar for all four drugs: the specificity of Kit-LPA ranged from 93.7\% (95\% CI 87-97\%) to 99.1\% (95\% CI 95-100\%) and for LPA from 94.5\% (95\% CI 88-99\%) to 100\% (95\% CI 97-100\%) (table 1). These results demonstrate that the use of Trans-Filter-extracted DNA enables similar test outcomes to the LPA for the rapid determination of drug resistance profiles (figure 3).

\section{Performance of Kit-LPA}

Using LPA as a gold standard, the Kit-LPA showed a sensitivity and specificity in the range of 96.5-100\% and 98.7-100\%, respectively, for all four drugs (table 2, 95\% CI values are included). There was no significant difference in the performance of Kit-LPA versus LPA ( $\mathrm{p}=0.48-1.0$ for all four drugs) and a concordance of $98.8-100 \%$ ( $\kappa$ value $0.83-1.0$ ) was noted (table 2). In case of discrepancy, both Kit-LPA and LPA were repeated.

In a stratified analysis of LPA results from 11 out of 16 children for whom these results were available, a concordance of 100\% ( $\kappa$ value 1.0) was noted between Kit-LPA and LPA.

\section{Comparative performance of Kit-LPA, LPA and Xpert MTB/RIF}

Kit-LPA and LPA had a concordance of $97.1 \%$ ( $\kappa$ value 0.85 ) and $98.2 \%$ ( $\kappa$ value 0.90 ), respectively, with Xpert for RIF resistance determination.

\section{Bio-safety assessment}

None of the sputum samples processed by the "Transport kit” were $M$. tuberculosis positive after 6 weeks of incubation of Trans-Filter inoculated in MGIT culture (figure 4). Efficient disinfection of sputum samples was achieved irrespective of the smear grade status of the sample (figure 4). These results indicated $100 \%$ success in disinfection of sputum samples by the "Transport kit".

\section{Discussion}

In resource-limited countries with a high TB burden, a simple, rapid and cost-effective method for sputum transport from DMCs and PHCs to NRLs/IRLs remains an immediate and unmet need to this day [3]. 

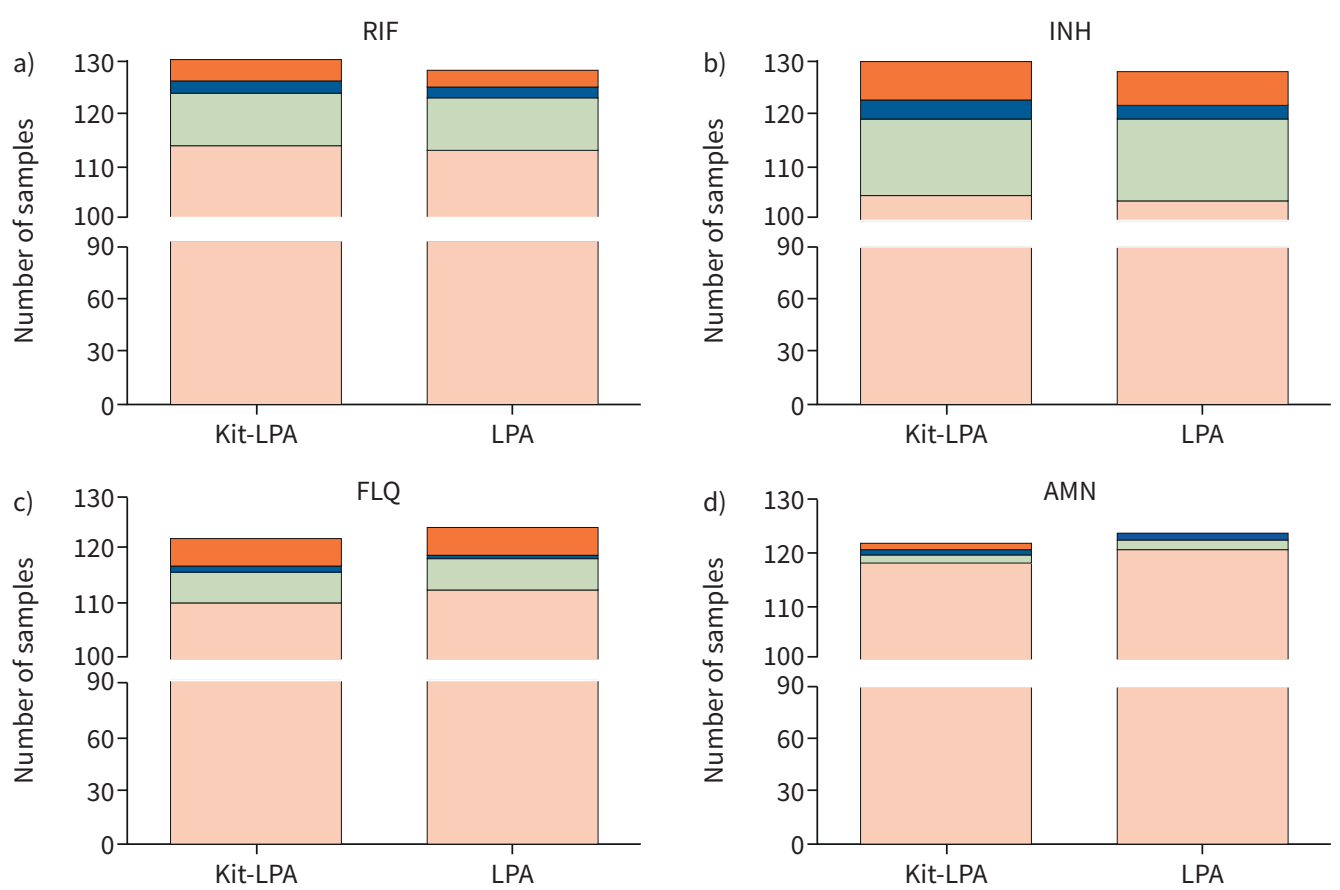

Genotypic wild type/phenotypic sensitive (TN) Genotypic mutant/phenotypic resistant (TP)

Genotypic wild type/phenotypic resistant (FN)

Genotypic mutant/phenotypic sensitive (FP)

FIGURE 3 Comparison of Kit-extracted DNA with line probe assay (Kit-LPA) and LPA versus Mycobacteria Growth Indicator Tube (MGIT)-drug susceptibility testing (DST) for detection of multidrug-resistant TB (MDR-TB) and extensively drug-resistant TB (XDR-TB). RIF: rifampicin; INH: isoniazid; FLQ: fluoroquinolone; AMN: aminoglycoside.

In India under NTEP, efforts are being made to link postal and courier services for sputum transport from peripheral centres to laboratories having culture and molecular diagnostics facilities to provide "Universal DST" services [3]. However, a requirement for triple-layer packaging, maintaining a cold chain during transport and speedy delivery are daunting challenges in the field [15].

In view of these challenges, the WHO's target product profile (TPP) has highlighted several desirable parameters for "sputum transport methods", which include compatibility with M. tuberculosis detection assays, a simple equipment-free procedure, bio-safety and stability during transportation [16]. To overcome the aforementioned challenges, we recently developed the "TB Concentration and Transport" kit for use at DMCs/PHCs to enable safe and ambient-temperature transport of sputum on Trans-Filter to a higher-level laboratory for further investigation. The Transport kit fulfils all the requirements of the TPP except for "compatibility with culture methods", which requires viable bacteria. In contrast, this kit fulfils the

TABLE 1 Performance of Kit-LPA and LPA versus MGIT-DST for the detection of MDR-TB and XDR-TB

\begin{tabular}{|c|c|c|c|c|c|c|c|}
\hline \multicolumn{4}{|c|}{ Kit-LPA versus MGIT-DST } & \multicolumn{4}{|c|}{ LPA versus MGIT-DST } \\
\hline $\operatorname{Drug}^{\#}(n)^{q}$ & $\begin{array}{c}\text { Sensitivity \% } \\
\left(95 \% \mathrm{Cl}^{+}\right.\end{array}$ & $\begin{array}{c}\text { Specificity \% } \\
(95 \% \mathrm{CI})\end{array}$ & $\begin{array}{c}\text { Concordance \% } \\
(\mathbf{( к})^{\S}\end{array}$ & Drug $^{\#}$ & $\begin{array}{c}\text { Sensitivity \% } \\
(95 \% \mathrm{CI})\end{array}$ & $\begin{array}{c}\text { Specificity \% } \\
(95 \% \mathrm{Cl})\end{array}$ & $\begin{array}{c}\text { Concordance \% } \\
\text { (к) }\end{array}$ \\
\hline $\operatorname{RIF}(n=130)$ & $83.3(52-98)$ & $96.6(91-99)$ & $95.3(0.74)$ & $\operatorname{RIF}(n=128)$ & $83.3(52-98)$ & 97.4 (93-99) & $96.1(0.77)$ \\
\hline INH $(n=130)$ & 77.7 (52-94) & $93.7(87-97)$ & $91.5(0.67)$ & INH $(n=128)$ & $83.3(59-96)$ & 94.5 (88-99) & $91.5(0.73)$ \\
\hline FLQ $(n=122)$ & $85.7(42-100)$ & 95.7 (90-99) & $95.1(0.64)$ & FLQ (n=124) & $85.7(42-100)$ & $95.7(90-99)$ & $95.1(0.64)$ \\
\hline AMN $(n=122)$ & 66.6 (9-99) & $99.1(95-100)$ & $98.3(0.66)$ & AMN $(n=124)$ & $66.6(9-99)$ & $100(97-100)$ & $99.2(0.79)$ \\
\hline
\end{tabular}

Kit-LPA: Kit-extracted DNA with line probe assay; LPA: line probe assay; MGIT-DST: Mycobacteria Growth Indicator Tube-drug susceptibility testing; MDR-TB: multidrug-resistant TB; XDR-TB: extensively drug-resistant TB. "RIF: rifampicin; INH: isoniazid; FLQ: fluoroquinolones; AMN: aminoglycosides. 'The details of samples are provided in supplementary figures S2 and S3. ${ }^{+} \mathrm{Cl}$ : confidence interval. ${ }^{\S} \mathrm{K}$ : Cohen's kappa coefficient. 
TABLE 2 Performance of Kit-LPA for the detection of MDR-TB and XDR-TB ${ }^{\#}$

\begin{tabular}{|c|c|c|c|c|}
\hline \multirow[b]{2}{*}{ Drug $^{\text {I }}$ (No. of samples) ${ }^{+}$} & \multirow[b]{2}{*}{ Gene target } & \multicolumn{3}{|c|}{ Kit-LPA versus LPA } \\
\hline & & Sensitivity $\%(95 \% \mathrm{Cl})^{\S}$ & Specificity $\%(95 \% \mathrm{CI})$ & Concordance $\%(\mathbf{k})^{f}$ \\
\hline RIF & $\operatorname{rpoB}(\mathrm{n}=197)$ & $100(85-100)$ & $98.8(96-100)$ & $98.8(0.95)$ \\
\hline \multirow[t]{2}{*}{ INH } & katG $(n=196)$ & $96.5(82-100)$ & $100(98-100)$ & $99.4(0.98)$ \\
\hline & $\operatorname{inh} A(n=197)$ & $100(40-100)$ & $100(98-100)$ & $100(1.0)$ \\
\hline \multirow[t]{2}{*}{ FLQ } & gyrA $(\mathrm{n}=179)$ & $100(80-100)$ & $98.7(96-100)$ & $98.8(0.94)$ \\
\hline & $\operatorname{gyrB}^{\# \#}(\mathrm{n}=179)$ & Not estimable & $100(98-100)$ & 100 (Not estimable) \\
\hline \multirow[t]{2}{*}{ AMN } & $\operatorname{rrs}(\mathrm{n}=179)$ & $100(48-100)$ & $98.8(96-100)$ & $98.8(0.83)$ \\
\hline & eis $(n=179)$ & $100(2-100)$ & $100(98-100)$ & $100(1.0)$ \\
\hline
\end{tabular}

Kit-LPA: Kit-extracted DNA with line probe assay; LPA: line probe assay; MDR-TB: multidrug-resistant TB; XDR-TB: extensively drug-resistant TB. \#Using LPA as a gold standard. "RIF: rifampicin; INH: isoniazid; FLQ: fluoroquinolones; AMN: aminoglycosides. "The details of samples are provided in supplementary figure S4. ${ }^{\S} \mathrm{Cl}$ : confidence interval. ${ }^{f} \mathrm{~K}$ : Cohen's kappa coefficient. ${ }^{\# \#}$ There was no mutant sample in gyrB drug target; therefore sensitivity and $\mathrm{\kappa}$ value of concordance could not be estimated.

bio-safety criterion. It achieved a minimum 8-log reduction of $M$. tuberculosis [6] thereby minimising exposure of workers to bio-hazards during processing and transportation of samples from DMCs/PHCs. The level of disinfection of $M$. tuberculosis provided was comparable to that provided by the "Sample Reagent” in the Xpert assay [6]. The Transport kit is compatible with Mol-DST approaches, such as DNA sequencing [6] and LPA (present study). In this study, the compatibility of the kit was assessed with the WHO-recommended LPA detection of RIF and INH resistance in place of phenotypic DST [4], and this points to the increasing scope of molecular DST tests to replace culture-based approaches in the near future.

A comparison of the features of our kit with those of other sputum transport kits currently available on the market [16] indicates our Transport kit to be more cost-effective (INR 100 or USD 1.40 per sample) than others, in addition to being bio-safe and compatible with dry sputum transport (table 3). The "TB DNA Extraction” kit is also attractively priced at INR 100 (USD 1.36) per sample as compared to the currently

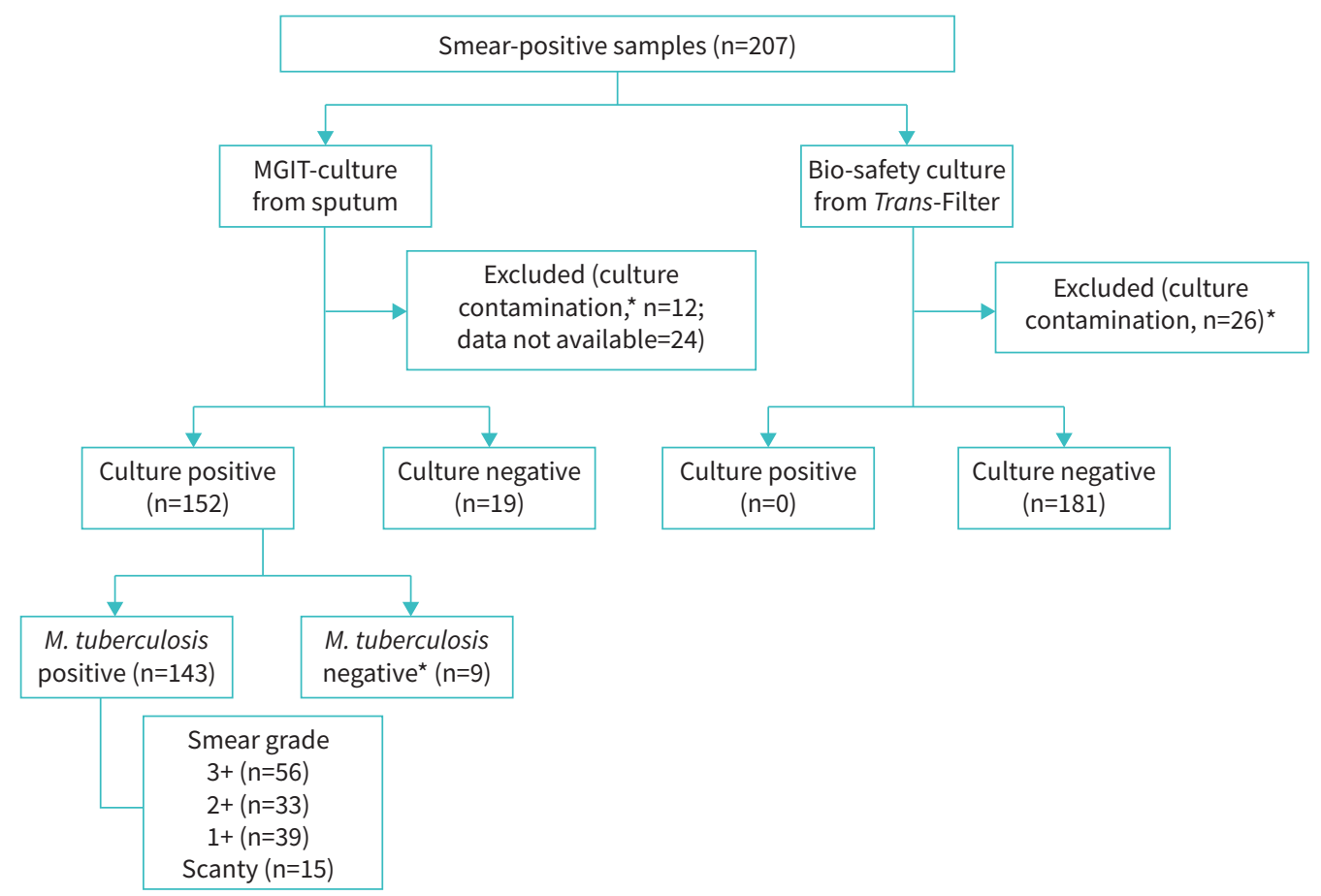

FIGURE 4 Bio-safety culture results of samples processed by the "TB Concentration \& Transport" kit. *Negative for Mycobacterium tuberculosis by SD Bioline MPT64 Ag Rapid test. MGIT: Mycobacteria Growth Indicator Tube. 


\begin{tabular}{|c|c|c|c|c|c|c|}
\hline $\begin{array}{l}\text { TB Concentration \& } \\
\text { Transport kit } \\
\text { (used in present } \\
\text { study) }\end{array}$ & $\begin{array}{l}\text { Advanced } \\
\text { Microdevices } \\
\text { (mdi), Ambala, } \\
\text { India }\end{array}$ & $\begin{array}{l}\text { Dried sputum on } \\
\text { filter }\end{array}$ & $\begin{array}{l}\text { NAATs (LPA ( present } \\
\text { study), PCR, Sanger } \\
\text { sequencing [6]) }\end{array}$ & $\begin{array}{l}\text { Spill proof, } \\
\text { ambient-temperature } \\
\text { transport, bio-safe }\end{array}$ & $\begin{array}{l}\text { Field testing } \\
\text { is pending }\end{array}$ & $\begin{array}{l}\text { INR } 100 / \\
\quad \text { USD } 1.36\end{array}$ \\
\hline $\begin{array}{l}\text { FTA card (recently } \\
\text { discontinued) }\end{array}$ & $\begin{array}{l}\text { Whatman, GE } \\
\text { Healthcare Life } \\
\text { Sciences, } \\
\text { Pittsburgh, PA, } \\
\text { USA }\end{array}$ & $\begin{array}{l}\text { Spotted sputum } \\
\text { on filter }\end{array}$ & $\begin{array}{l}\text { NAATs (LPA, PCR [31, } \\
\text { 32]) }\end{array}$ & $\begin{array}{l}\text { Spill proof, } \\
\text { ambient-temperature } \\
\text { transport }\end{array}$ & Not bio-safe & $\begin{array}{l}\text { INR } 165 / \\
\quad \text { USD } 2.25\end{array}$ \\
\hline
\end{tabular}

used kit in LPA, i.e. GenoLyse ${ }^{\circledR}$ DNA Extraction kit (INR 138 or USD 1.88/sample) and other commercially available kits for DNA isolation such as QIAamp DNA Mini Kit (Qiagen, USA, INR 191 or USD 2.60/sample) and PrimeXtract extraction kit (Long Horn Vaccines and Diagnostics, USA, INR 262 or USD 3.57/sample).

The sensitivity and specificity of Kit-LPA for RIF, INH, FLQ and AMN were in the range of 66.6-85.7\% and 93.7-99.1\%, respectively, which was quite similar to that of LPA using MGIT-DST as a reference standard (table 1), with a concordance value of 91.5-98.3\% ( $\kappa$ value 0.64-0.74) for MDR-TB and XDR-TB (table 1). The sensitivity of LPA (both Kit-LPA and LPA) for detecting drug resistance in our study $(\sim 67 \%$ to $\sim 86 \%$ ) was somewhat lower than the pooled sensitivity reported previously in a meta-analysis (86-96\%) [4]. A possible reason for this lower sensitivity may be a comparatively smaller sample size, where a small discrepancy in classifying a sample as sensitive or resistant, results in a greater difference in sensitivity. The discrepancies noted between MGIT-DST and LPA/Kit-LPA results are summarised in supplementary table S2 and might be attributed either to the presence of mutations outside the resistance-determining region probed in LPA [17-19], or disputed or inferred mutations (which show low-level resistance in MGIT-DST) [20, 21], or heteroresistance as reported earlier [6, 19, 22].

The most important finding of this study was that the overall performance of Kit-LPA was quite similar to that of LPA (figure 3), and it is noteworthy that a minimal level of discordance was observed between these two tests ( $n=3$, supplementary table S2). The discrepancies were rechecked by repeating Kit-LPA and LPA tests. The interpretation of LPA as per manufacturer's instructions is based on the detection of band intensity (wild-type or mutant probe) being greater than or equal to that of the "Amplification Control" band [9, 10]. A slight difference in band intensity between Kit-LPA and LPA could have caused a difference in interpretation, thereby leading to discordance.

The feedback from the scientists and laboratory technicians revealed the biggest benefits of kits to be their ease-of-use, bio-safe sputum transport on filter paper at ambient temperature and easy integration of Kit-extracted DNA with LPA protocol. The feedback also highlighted the minimal training required by laboratory technicians for the Transport kit procedure. This feedback will be helpful for assessing these kits in a feasibility study under field settings in the future.

\section{Strengths and limitations of the study}

We have demonstrated in the present study that DNA extracted from Trans-Filter is compatible with first line and second line LPA for the detection of MDR-TB and XDR-TB. These findings are especially 
noteworthy when seen in the context of the current scenario of NTEP's DST programme, where Hain's LPA is used for detection of MDR-TB and XDR-TB (depending on RIF susceptibility result) for all TB cases diagnosed by Xpert. Mol-DST tests require sophisticated laboratory infrastructure with bio-safety compliance, and they are not easy to implement at the peripheral level. The bio-safe sputum transport kit reported here is highly suitable for use in lower-level set ups such as DMCs and PHCs, as it eliminates the use of equipment for concentrating sputum and minimises the risk of aerosol generation. The transport filter combines smoothly with the "TB DNA Extraction” kit to provide pure M. tuberculosis DNA from sputum for integration with various molecular DST tests. The second noteworthy finding was that the performance of Kit-LPA was highly concordant with LPA for all four drugs and with Xpert for determining RIF resistance. Thirdly, the feedback from laboratory technicians and scientists highlighted the benefit of Trans-Filter for bio-safe dried sputum transport and integration of extracted DNA with LPA. A limitation of the study was that sputum Trans-Filters were not transported from remote areas.

\section{Conclusions}

Our findings have laid the foundation for the use of these kits in achieving the goal of "Universal DST". Owing to its ease of use, cost-effectiveness and patient accessibility, the sputum processing and transport technology described here has the potential to transform the diagnostic supply chain by providing near-patient cost-effective "Universal DST" services to TB subjects residing in remote geographical areas of India. This technology has the potential to positively impact DST not only in India, but also in other high-burden countries where sample transportation is a formidable barrier to the widespread implementation of DST. Kit-LPA is poised for evaluation in field settings under NTEP for the detection of MDR-TB and XDR-TB.

Acknowledgements: We acknowledge the technical assistance of Gavish Kumar, Grish Sah, Kamaaluddin, Mehreen and Rinku Mishra at the NITRD site, and Abhinav Goyal from Advanced Microdevices. We also acknowledge all the enrolled patients for providing consent to participate in the study.

Support statement: This study was funded by India Tuberculosis Research Consortium (ITRC) of the Indian Council of Medical Research, Government of India (grant number 5/8/5/ITRC/7/2018/ECD-I) received jointly by J. Sivaswami Tyagi, N.K. Gupta, S. Haldar, V.P. Myeendu and R. Sarin. J. Sivaswami Tyagi is thankful to the National Academy of Sciences (India) for a Senior Scientist Fellowship. The fellowships of D. Anthwal and R.K. Gupta were from the ITRC grant awarded to S. Haldar. Funding information for this article has been deposited with the Crossref Funder Registry.

Conflict of interest: D. Anthwal reports personal fees from the ICMR (fellowship support) during the conduct of the study and a patent, "Apparatus and method for processing a sample for rapid diagnosis of tuberculosis and safe transport of bacteria" (patent application number 201811042155), pending. R.K. Gupta has a patent, "Apparatus and method for processing a sample for rapid diagnosis of tuberculosis and safe transport of bacteria" (patent application number 201811042155), pending; and manufactured and provided the TB Concentration \& Transport and TB DNA Extraction kits, and participated in training of the laboratory staff who used the kit. R. Singhal reports grants from the ICMR during the conduct of the study. M. Bhalla reports grants from the ICMR during the conduct of the study. A.K. Verma reports grants from the ICMR during the conduct of the study and a patent, "Apparatus and method for processing a sample for rapid diagnosis of tuberculosis and safe transport of bacteria" (patent application number 201811042155), pending. K.U. Khayyam reports grants from the ICMR during the conduct of the study. C.P. Myneedu reports grants from the ICMR during the conduct of the study and a patent, "Apparatus and method for processing a sample for rapid diagnosis of tuberculosis and safe transport of bacteria" (patent application number 201811042155), pending. R. Sarin reports grants from the ICMR during the conduct of the study and a patent, "Apparatus and method for processing a sample for rapid diagnosis of tuberculosis and safe transport of bacteria" (patent application number 201811042155), pending. A. Gupta has a patent, "Apparatus and method for processing a sample for rapid diagnosis of tuberculosis and safe transport of bacteria" (patent application number 201811042155), pending; and manufactured and provided the TB Concentration \& Transport and TB DNA Extraction kits, and participated in training of the laboratory staff who used the kit. N.K. Gupta has a patent, "Apparatus and method for processing a sample for rapid diagnosis of tuberculosis and safe transport of bacteria" (patent application number 201811042155), pending; and manufactured and provided the TB Concentration \& Transport and TB DNA Extraction kits, and participated in training of the laboratory staff who used the kit. M. Singh has nothing to disclose. J. Sivaswami Tyagi reports grants from ICMR during the conduct of the study and a patent, "Apparatus and method for processing a sample for rapid diagnosis of tuberculosis and safe transport of bacteria" (patent application number 201811042155), pending. S. Haldar reports grants from the ICMR during the conduct of the study and a patent, "Apparatus and method for processing a sample for rapid diagnosis of tuberculosis and safe transport of bacteria" (patent application number 201811042155), pending. 
References

1 WHO. Global Tuberculosis Report. Geneva, World Health Organization, 2020.

2 Behra D. TB control in India in the COVID era. Ind J Tuberculosis 2021; 68: 128-133.

3 NTEP. India TB Report-National Tuberculosis Elimination Programme Annual Report. India, National Tuberculosis Elimination Programme, 2020.

4 WHO. Rapid Diagnostics for Tuberculosis Detection: WHO Consolidated Guidelines on Tuberculosis. Geneva, World Health Organization, 2020.

5 RNTCP. Guidelines on Programmatic Management of Drug Resistant TB (PMDT) in India. India, Revised National TB Control Program, 2017.

6 Anthwal D, Lavania S, Gupta RK, et al. Development and evaluation of novel bio-safe filter paper-based kits for sputum microscopy and transport to directly detect Mycobacterium tuberculosis and associated drug resistance. PLOS ONE 2019; 14: e0220967.

7 RNTCP. Technical and Operational Guidelines of Tuberculosis Control in India. India, Revised National TB Control Program, 2016.

8 Global Laboratory Initiative. Stop TB Partnership. Mycobacteriology Laboratory Manual. First Edition. 2014.

9 Hain-Lifesciences. GenoType MTBDRplus VER 2.0: Molecular Genetic Assay for Identification of the M. tuberculosis complex and its Resistance to Rifampicin and Isoniazid from Clinical Specimens and Cultivated Samples. Nehren, Germany, Hain-Lifesciences, 2012.

10 Hain-Lifesciences. GenoType MTBDRsI VER 2.0: Molecular Genetic Assay for Identification of the M. tuberculosis Complex and its Resistance to Fluoroquinolones and Aminoglycosides/Cyclic Peptides from Sputum Specimens or Cultivated Samples. Nehren, Germany, Hain-Lifesciences, 2015.

11 Cepheid. Xpert MTB/RIF Manual. Sunnyvale, CA, Cepheid, 2012.

12 WHO. Technical Manual for Drug Susceptibility Testing of Medicines Used in the Treatment of Tuberculosis. Geneva, World Health Organization, 2018.

13 WHO. Technical Report on Critical Concentrations for Drug Susceptibility Testing of Medicines used in the Treatment of Drug-Resistant Tuberculosis. Geneva, World Health Organization, 2018.

14 Faul F, Erdfelder E, Lang AG, et al. G*Power 3: a flexible statistical power analysis program for the social, behavioral, and biomedical sciences. Behav Res Methods 2007; 39: 175-191.

15 RNTCP. National Strategic Plan for Tuberculosis Elimination 2017-2025. India, Revised National TB Control Program, 2017.

16 WHO. Technical Expert Group Meeting Report: Commercial Products for Preserving Clinical Specimens for the Diagnosis of Tuberculosis. Geneva, World Health Organization, 2017.

17 Georghiou SB, Magana M, Garfein RS, et al. Evaluation of genetic mutations associated with Mycobacterium tuberculosis resistance to amikacin, kanamycin and capreomycin: a systematic review. PLoS ONE 2012; 7: e33275.

18 Seifert M, Catanzaro D, Catanzaro A, et al. Genetic mutations associated with isoniazid resistance in Mycobacterium tuberculosis: a systematic review. PLOS ONE 2015; 10: e0119628.

19 Miotto P, Piana F, Penati V, et al. Use of genotype MTBDR assay for molecular detection of rifampin and isoniazid resistance in Mycobacterium tuberculosis clinical strains isolated in Italy. J Clin Microbiol 2006; 44: 2485-2491.

20 Miotto P, Cabibbe AM, Borroni E, et al. Role of disputed mutations in the rpoB gene in interpretation of automated liquid MGIT culture results for Rifampin susceptibility testing of Mycobacterium tuberculosis. $J$ Clin Microbiol 2018; 56: e01599-17.

21 Singhal R, Anthwal D, Kumar G, et al. Genotypic characterization of 'inferred' rifampin mutations in GenoType MTBDRplus assay and its association with phenotypic susceptibility testing of Mycobacterium tuberculosis. Diagn Microbiol Infect Dis 2020; 96: 114995.

22 Tolani MP, D'Souza D T, Mistry NF. Drug resistance mutations and heteroresistance detected using the GenoType MTBDRplus assay and their implication for treatment outcomes in patients from Mumbai, India. BMC Infect Dis 2012; 12: 9.

23 Omar SV, Peters RP, Ismail NA, et al. Field evaluation of a novel preservation medium to transport sputum specimens for molecular detection of Mycobacterium tuberculosis in a rural African setting. Trop Med Int Health 2016; 21: 776-782.

24 Osei Sekyere J, Maphalala N, Malinga LA, et al. A comparative evaluation of the new genexpert MTB/RIF ultra and other rapid diagnostic assays for detecting tuberculosis in pulmonary and extra pulmonary specimens. Sci Rep 2019; 9: 16587.

25 Daum LT, Choi Y, Worthy SA, et al. A molecular transport medium for collection, inactivation, transport, and detection of Mycobacterium tuberculosis. Int J Tuberc Lung Dis 2014; 18: 847-849.

26 Daum LT, Fourie PB, Bhattacharyya S, et al. Next-generation sequencing for identifying pyrazinamide resistance in Mycobacterium tuberculosis. Clin Infect Dis 2014; 58: 903-904.

27 Daum LT, Konstantynovska OS, Solodiankin OS, et al. Next-generation sequencing for characterizing drug resistance-conferring Mycobacterium tuberculosis genes from clinical isolates in the Ukraine. J Clin Microbiol 2018; 56: e00009-18. 
28 Daum LT, Konstantynovska OS, Solodiankin OS, et al. Characterization of novel Mycobacterium tuberculosis pncA gene mutations in clinical isolates from the Ukraine. Diagn Microbiol Infect Dis 2019; 93: 334-338.

29 Daum LT, Fischer GW, Sromek J, et al. Characterization of multi-drug resistant Mycobacterium tuberculosis from immigrants residing in the USA using Ion Torrent full-gene sequencing. Epidemiol Infect 2014; 142: 1328-1333.

30 Sharma S, Ryndak MB, Aggarwal AN, et al. Transcriptome analysis of mycobacteria in sputum samples of pulmonary tuberculosis patients. PLOS ONE 2017; 12: e0173508.

31 Rabodoarivelo MS, Imperiale B, Andrianiavomikotroka R, et al. Performance of four transport and storage systems for molecular detection of multidrug-resistant tuberculosis. PLOS ONE 2015; 10: e0139382.

32 Reeve BWP, McFall SM, Song R, et al. Commercial products to preserve specimens for tuberculosis diagnosis: a systematic review. Int J Tuberc Lung Dis 2018; 22: 741-753. 